TRANSACTIONS OF THE

AMERICAN MATHEMATICAL SOCIETY

Volume 356, Number 2, Pages 691-702

S 0002-9947(03)03441-X

Article electronically published on September 22, 2003

\title{
ON A CONJECTURE OF WHITTAKER CONCERNING UNIFORMIZATION OF HYPERELLIPTIC CURVES
}

\author{
ERNESTO GIRONDO AND GABINO GONZÁLEZ-DIEZ
}

\begin{abstract}
This article concerns an old conjecture due to E. T. Whittaker, aiming to describe the group uniformizing an arbitrary hyperelliptic Riemann surface $y^{2}=\prod_{i=1}^{2 g+2}\left(x-a_{i}\right)$ as an index two subgroup of the monodromy group of an explicit second order linear differential equation with singularities at the values $a_{i}$.

Whittaker and collaborators in the thirties, and R. Rankin some twenty years later, were able to prove the conjecture for several families of hyperelliptic surfaces, characterized by the fact that they admit a large group of symmetries. However, general results of the analytic theory of moduli of Riemann surfaces, developed later, imply that Whittaker's conjecture cannot be true in its full generality.

Recently, numerical computations have shown that Whittaker's prediction is incorrect for random surfaces, and in fact it has been conjectured that it only holds for the known cases of surfaces with a large group of automorphisms.

The main goal of this paper is to prove that having many automorphisms is not a necessary condition for a surface to satisfy Whittaker's conjecture.
\end{abstract}

\section{INTRODUCTION}

One of the foundational results in Riemann surface theory is that a compact Riemann surface can be viewed either as an algebraic curve $F(x, y)=0$, or as the quotient space of the unit disc $\mathbb{D}$ by the action of a Fuchsian group. However, this correspondence can be made explicit only in some exceptional cases (see, e.g., [2]).

E. T. Whittaker, following ideas of H. Poincaré, put forward the following strategy to understand this correspondence in the hyperelliptic case:

Every hyperelliptic surface $C$ of genus $g$ greater than one, arises from a set of $2 g+2$ points $A=\left\{a_{1}, \ldots, a_{2 g+2}\right\} \subset \widehat{\mathbb{C}}:=\mathbb{C} \cup\{\infty\}$ as an algebraic curve

$$
y^{2}=f(x), \quad \text { where } f(x):=\prod_{i=1}^{N}\left(x-a_{i}\right),
$$

with $N=2 g+2$ if $A \subset \mathbb{C}$, and $N=2 g+1$ if, say, $a_{2 g+2}=\infty$.

The hyperelliptic involution and the hyperelliptic function $C \stackrel{\pi}{\longrightarrow} \frac{C}{\langle J\rangle} \simeq \widehat{\mathbb{C}}$ are given by $J(x, y)=(x,-y)$ and $\pi(x, y)=x$ respectively.

Received by the editors July 23, 2002.

2000 Mathematics Subject Classification. Primary 30F10; Secondary 14H15.

Key words and phrases. Accessory parameters, Schwarzian derivative, uniformization of Riemann surfaces, hyperelliptic curves.

Both authors were supported in part by Grant BFM2000-0031, DGI.MCYT. 
From the point of view of uniformization, there is a torsion-free Fuchsian group $K$ such that the universal covering map $p: \mathbb{D} \rightarrow C$ is represented by the obvious projection $\mathbb{D} \rightarrow \mathbb{D} / K \simeq C$.

Likewise, the map $X:=\pi \circ p: \mathbb{D} \longrightarrow \widehat{\mathbb{C}}$ corresponds to a projection of the form $\mathbb{D} \longrightarrow \mathbb{D} / \Gamma \simeq \widehat{\mathbb{C}}$, where $\Gamma$ is a Fuchsian group generated by $2 g+2$ order-two elements, say $\gamma_{1}, \ldots, \gamma_{2 g+2}$. The group $K$ is then the subgroup generated by the transformations $\gamma_{i} \gamma_{j}$.

Now the fundamental fact is that $\Gamma$, the covering group of the map $X: \mathbb{D} \longrightarrow \widehat{\mathbb{C}}$, is precisely the monodromy group of the second order Fuchsian differential equation

$$
y^{\prime \prime}(z)+\frac{1}{2}\left(S\left(X^{-1}\right)(z)\right) y(z)=0,
$$

where $S\left(X^{-1}\right)(z)$ is a rational function called the Schwarzian derivative of the multivalued function $X^{-1}$ (see definition below). Thus, a link between the algebraic object, the curve, and the transcendental one, the group $K$, would have been established if we could find an expression for $S\left(X^{-1}\right)(z)$ in terms of the parameters defining the algebraic equation, namely $a_{1}, \ldots, a_{2 g+2}$.

In 1929 [19] E.T. Whittaker conjectured that, for a hyperelliptic curve given by equation (1.1), we have

$$
S\left(X^{-1}\right)(z)=\frac{3}{8}\left(\left(\frac{f^{\prime}(z)}{f(z)}\right)^{2}-\frac{2 g+2}{2 g+1} \frac{f^{\prime \prime}(z)}{f(z)}\right) .
$$

Before we proceed any further, let us say that it is now known that Whittaker's conjecture cannot hold in its full generality. In fact, using the tools of the theory of Teichmüller spaces that was developed later, I. Kra [14] was able to prove that the coefficients of the rational function $S\left(X^{-1}\right)(z)$ do not depend holomorphically on the parameters $a_{k}$, whereas those of the right hand side of equation (1.2) obviously do.

As far as we know, the history of this conjecture goes as follows:

It was early noticed that the analogous statement for genus one surfaces holds, as can be seen after some manipulation with Weierstrass' $\wp$ function (see [21, p.439). The first curve of higher genus for which E.T. Whittaker checked equation (1.2), in his 1929 paper [19] was the genus two curve $y^{2}=x^{5}+1$. This result was extended one year later by M. Mursi [15] to the curve $y^{2}=x^{7}+1$, of genus three, and to all curves of the form $y^{2}=x^{2 g+1}+1$ by S.C. Dahr [6] in 1935. Other works on Whittaker's conjecture carried out about the same time are due to D.P. Dalzell [5], J. Hodgkinson [13] and Whittaker's son J.M. Whittaker [20]. It was not until some twenty years later that R.A. Rankin [16] substantially enlarged this list by proving relation (1.2) for a collection of curves "whose branch points", the values $a_{k}$, "form sets possessing certain symmetrical properties" in the author's own words. The conjecture was taken up by D.V. Chudnovsky and G.V Chudnovsky in their 1990 paper [4]. There, they performed a number of numerical experiments to find that equation (1.2) is incorrect for random surfaces. So it was natural to conjecture ([4], pp. 143 and 148) that the only curves for which Whittaker's prediction holds are those encountered by Rankin.

The aim of this paper is to prove that there are curves beyond those listed by Rankin for which Whittaker's conjecture holds (Theorem 4.8). The problem of characterizing the hyperelliptic surfaces that satisfy the conjecture remains open. 


\section{WhitTAKER'S CONJECTURE}

Definition 2.1. The Schwarzian derivative of a holomorphic function $\phi$, denoted $S(\phi)$, is given by

$$
S(\phi)(z)=\frac{\phi^{\prime \prime \prime}(z)}{\phi^{\prime}(z)}-\frac{3}{2}\left(\frac{\phi^{\prime \prime}(z)}{\phi^{\prime}(z)}\right)^{2} .
$$

The relevance of this differential operator relies on the following two properties:

$$
\begin{aligned}
& S(\phi) \equiv 0 \Leftrightarrow \phi \text { is a Möbius transformation, } \\
& S(\phi \circ \alpha)(z)=\alpha^{\prime}(z)^{2} S(\phi)(\alpha(z))+S(\alpha)(z) .
\end{aligned}
$$

Recall that a regular, or normal, cover $X: \mathbb{D} \rightarrow \widehat{\mathbb{C}}$ ramified over a set of values $A=\left\{a_{1}, \ldots, a_{n}\right\} \subset \widehat{\mathbb{C}}(n \geq 3)$ with branching indices $\nu_{1}, \ldots, \nu_{n}$, is a surjective holomorphic map ramified (only) over the set $A$, and such that the branching order at each point in the fibre of $a_{k}$ is $\nu_{k}$. It is a well known fact that being a regular cover is the same as saying that the map $X$ is equivalent to a quotient map $\mathbb{D} \longrightarrow \mathbb{D} / \Gamma$, where $\Gamma$ is the group of covering transformations of $X$. The group $\Gamma$ is a Fuchsian group, said to have signature $\left(0 ; \nu_{1}, \ldots, \nu_{n}\right)$.

It is also known that if this signature is hyperbolic, that is, $\sum_{j=1}^{n}\left(1-\nu_{j}^{-1}\right)>2$, then a regular cover with given branching data $\left(\left\{a_{k}\right\},\left\{\nu_{k}\right\}\right)$ always exists, and two such maps differ by pre-composition with an automorphism $T$ of $\mathbb{D}$ (see, e.g., [8], p. 219). In particular, we can apply properties (2.1) and (2.2) to infer that the Schwarzian derivative $S\left(X^{-1}\right)(z)$ depends neither on the choice of the covering map $X$, nor on that of a particular branch of its inverse map. In other words, it only depends on the branching data (cf. [14], (4.1)).

Definition 2.2. Given a set of $2 g+2$ distinct points $A=\left\{a_{1}, \ldots, a_{2 g+2}\right\} \subset \widehat{\mathbb{C}}$, with $g \geq 2$, we shall denote by $C_{A}$ the algebraic curve given by equation (1.1), by $p: \mathbb{D} \rightarrow C_{A}$ a uniformizing map, and by $\pi: C_{A} \rightarrow \widehat{\mathbb{C}}$ the hyperelliptic map $\pi(x, y)=x$. Let $X: \mathbb{D} \longrightarrow \widehat{\mathbb{C}}$ be given by $X=\pi \circ p$; this is a regular cover of signature $\left(0 ; 2,{ }^{2 g+2}, 2\right)$. The Schwarzian derivative of $X^{-1}$, which, by the above comments, depends only on $A$ and not on the choice of $p: \mathbb{D} \rightarrow C_{A}$, will be denoted by $S_{A}$, namely

$$
S_{A}(z):=S\left(X^{-1}\right)(z)=\frac{\left(X^{-1}\right)^{\prime \prime \prime}(z)}{\left(X^{-1}\right)^{\prime}(z)}-\frac{3}{2}\left(\frac{\left(X^{-1}\right)^{\prime \prime}(z)}{\left(X^{-1}\right)^{\prime}(z)}\right)^{2} .
$$

By examining the local behaviour at the branch points, it can be shown (see [16], p. 37) that

$$
S_{A}(z)=\frac{3}{8}\left(\sum_{i=1}^{2 g+2} \frac{1}{\left(z-a_{i}\right)^{2}}+\frac{h(z)}{f(z)}\right),
$$

where $f(z)$ is given by equation (1.1), and $h(z)$ is a certain polynomial of the form

$$
h(z)=-(2 g+2) z^{2 g}+2 g\left(\sum_{i=1}^{2 g+2} a_{i}\right) z^{2 g-1}+c_{2 g-2} z^{2 g-2}+\cdots+c_{1} z+c_{0} .
$$

The $2 g-1$ coefficients $c_{0}, c_{1}, \ldots, c_{2 g-2}$ are clasically known as the accessory parameters. It should be said that some authors define the accessory parameters in a slightly different way (see, e.g., [14]), but it is always an easy matter to establish the relation between the various definitions. 
Definition 2.3. The rational function

$$
W_{A}(z)=\frac{3}{8}\left(\left(\frac{f^{\prime}(z)}{f(z)}\right)^{2}-\frac{2 g+2}{2 g+1} \frac{f^{\prime \prime}(z)}{f(z)}\right)
$$

or, equivalently,

$$
W_{A}(z)=\frac{3}{8}\left(\sum_{i} \frac{1}{\left(z-a_{i}\right)^{2}}-\frac{1}{2 g+1} \frac{f^{\prime \prime}(z)}{f(z)}\right)
$$

will be referred to as the Whittaker rational function associated to the curve $C_{A}$.

Now we can state the conjecture.

Whittaker's Conjecture ([19], p. 275). For any set $A$ of $2 g+2$ different points, the identity

$$
S_{A} \equiv W_{A}
$$

holds.

Along with the discovery of the truth of the conjecture for the cases referred to in Section 1, one of the reasons that might have led Whittaker to state his conjecture is that the Whittaker rational function enjoys the same quadratic-like behaviour as the Schwarzian derivative. Namely,

Proposition 2.4. For any Möbius transformation $T$ we have

$$
S_{A}(z)=T^{\prime}(z)^{2} S_{T(A)}(T(z)),
$$

and

$$
W_{A}(z)=T^{\prime}(z)^{2} W_{T(A)}(T(z)) .
$$

Proof. The first equality is obtained from formula (2.2) by replacing $\phi$ with $X^{-1}$, $\alpha$ with $T^{-1}$, and $z$ with $T(z)$.

The second one can be found in [16, p. 41.

Remark 2.5. From now on, we can restrict ourselves to the case in which all branch values are finite, that is, $A \subset \mathbb{C}$. This is permitted because, if this were not the case, we could bring the problem to this situation by applying a suitable Möbius transformation $T$. By virtue of Proposition 2.4, this will not affect the truth of the conjecture (see [16], p. 40).

Following Rankin, we now consider the difference

$$
Q_{A}(z)=S_{A}(z)-W_{A}(z) .
$$

Comparing the second expression for $W_{A}(z)$ given in Definition 2.3 with the identity (2.3), we infer that $Q_{A}(z)=p(z) / f(z)$, where $p(z)$ is a polynomial with $\operatorname{deg}(p) \leq 2 g-2$.

There is a meromorphic quadratic differential $\omega_{A}$ on $C_{A}$ associated to the rational function $Q_{A}(z)$. It is defined as follows:

Definition 2.6. We will denote by $\omega_{A}$ the differential

$$
\omega_{A}=\pi^{*}\left(Q_{A}(z)(d z)^{2}\right),
$$

where $\pi: C_{A} \rightarrow \widehat{\mathbb{C}}$ is the hyperelliptic map $\pi(x, y)=x$.

Thus $\omega_{A}=Q_{A}(x) d x^{2}=\frac{p(x)}{f(x)} d x^{2}$, with $\operatorname{deg}(p) \leq 2 g-2$. 
Proposition 2.7. The meromorphic quadratic differential $\omega_{A}$ is in fact holomorphic and invariant with respect to the action of the automorphism group $\operatorname{Aut}\left(C_{A}\right)$.

Proof. The first statement follows from an easy computation of the local expression of $\omega_{A}$ near the poles of $Q_{A}$, similar to that carried out in a more general setting in 8], p. 254.

In order to prove that $\omega_{A}$ is $\operatorname{Aut}\left(C_{A}\right)$-invariant, we recall that every automorphism of a hyperelliptic surface commutes with the hyperelliptic involution. Therefore, each element $T$ of $\frac{\operatorname{Aut}\left(C_{A}\right)}{\langle J\rangle}$ acts on $\frac{C_{A}}{\langle J\rangle} \simeq \widehat{\mathbb{C}}$ as a Möbius transformation having the property that $T(A)=A$. Now, Proposition 2.4 implies that $Q_{A}(z)(d z)^{2}$ is $\frac{\operatorname{Aut}\left(C_{A}\right)}{\langle J\rangle}$-invariant; hence $\omega_{A}$ is $\operatorname{Aut}\left(C_{A}\right)$-invariant.

In terms of the quadratic differential $\omega_{A}$ the conjecture reads as follows:

Proposition 2.8. Whittaker's Conjecture holds if and only if $\omega_{A} \equiv 0$.

For the purpose of this article, the term Whittaker surface will refer to a compact hyperelliptic surface satisfying Whittaker's conjecture.

\section{Surfaces With MANY AUtomorphisms}

Definition 3.1. A surface with many automorphisms is a compact Riemann surface $C$ of genus greater than one such that the quotient space $\frac{C}{\operatorname{Aut}(C)}$ is isomorphic to the Riemann sphere $\widehat{\mathbb{C}}$ and, moreover, the projection $C \longrightarrow \frac{C}{\operatorname{Aut}(C)}$ ramifies over three values.

We have

Theorem 3.2. Every hyperelliptic surface $C$ with many automorphisms is a Whittaker surface.

Proof. The result follows from Proposition 2.7 once one notices that the dimension of the space of quadratic differentials that are $\operatorname{Aut}(C)$-invariant equals $3 \tilde{g}-3+n$, where $\tilde{g}$ is the genus of the quotient surface $\frac{C}{\operatorname{Aut}(C)}$ and $n$ is the number of branch values of the projection $C \longrightarrow \frac{C}{\operatorname{Aut}(C)}$ (see [8]). In our case this dimension equals zero, hence $\omega_{A} \equiv 0$.

Concerning Theorem 3.2, the following should be noted:

In 16, Rankin produced a list of explicit families of algebraic curves for which he could prove that the conjecture holds. This list includes all cases previously known up to then (and, in a sense, up to now, since our existence Theorem 4.8 does not provide an explicit example). It can be easily checked that all of them are surfaces with many automorphisms. In fact, more recent knowledge of the automorphism group of hyperelliptic surfaces (see, e.g., [3], 1]) would enable one to show that his list agrees with that of all the hyperelliptic surfaces with many automorphisms. Thus, from that point of view, the above result can be seen as a restatement of Theorem 3 in [16], the main difference being that our approach enables us to consider all surfaces with many automorphisms simultaneously, instead of studying each family separately. 


\section{The family of CuRVes $y^{2}=\left(x^{n}-1\right)\left(x^{n}-\lambda\right)$}

In this section we study the 1-parameter family of hyperelliptic curves of genus $n-1$

$$
C_{\lambda, n}=\left\{y^{2}=\left(x^{n}-1\right)\left(x^{n}-\lambda\right)\right\}, \quad \lambda \in \mathbb{R} \backslash\{0,1\}, \quad n \geq 3,
$$

whose branching locus is the set $A_{\lambda, n}:=\left\{1, \ldots, \xi_{n}^{n-1}, \lambda^{1 / n}, \ldots, \lambda^{1 / n} \xi_{n}^{n-1}\right\}$, with $\xi_{n}=\exp \left(\frac{2 \pi i}{n}\right)$.

Our goal is to show that there are curves in this family which do not have many automorphisms but that, nevertheless, are Whittaker surfaces.

Proposition 4.1. The group of automorphisms of the curve $C_{\lambda, n}$ is as follows:

i) If $\lambda \neq-1$, Aut $\left(C_{\lambda, n}\right) \equiv \mathbb{Z}_{2} \times D_{n}$ with generators $J(x, y)=(x,-y), \beta(x, y)=$ $\left(\lambda^{1 / n} / x, \lambda^{1 / 2} y / x^{n}\right)$ and $\sigma_{n}(x, y)=\left(\xi_{n} x, y\right)$.

ii) If $\lambda=-1$, the automorphism group contains also the order $2 n$ rotation $\sigma_{2 n}$. This is the only curve of the family which has many automorphisms.

Proof. The canonical projection

$$
C_{\lambda, n} \stackrel{\pi_{\lambda}}{\longrightarrow} \frac{C_{\lambda, n}}{\left\langle J, \beta, \sigma_{n}\right\rangle}
$$

is easily seen to be the sphere with four branch values of orders $2,2,2, n$.

Now suppose $C_{\lambda, n}$ has an extra automorphism $\tau$. Then, arguing as in the proof of Proposition 2.7, we see that $\tau$ induces an automorphism $T$ on $\frac{C_{\lambda, n}}{\langle J\rangle} \simeq \widehat{\mathbb{C}}$ that preserves the set $A_{\lambda, n}$. Therefore, $T$ has finite order.

It is not difficult to see that the Möbius transformation $T$ either interchanges the circles $|z|=1$ and $|z|=\left|\lambda^{1 / n}\right|$ or preserves each of them. Moreover, if $T$ is in the first case, then the Möbius transformation induced by $\beta \circ \tau$ will be in the second one. Thus, we can simply assume that $T$ preserves each of the circles and also the set $A_{\lambda, n}$, which in turn implies that it is a rotation fixing the origin. This is only possible if $T$ is a power of $\sigma_{n}$ when $\lambda \neq-1$, and a power of $\sigma_{2 n}$ when $\lambda=-1$.

Indeed, for $\lambda=-1$ we get the curve $y^{2}=\left(x^{2 n}-1\right)$. This curve admits the extra automorphism $\sigma_{2 n}(x, y)=\left(\xi_{2 n} x, y\right)$, which makes of it a surface with many automorphisms.

Recall that we had

$$
\omega_{\lambda, n}:=\omega_{A_{\lambda, n}}=\frac{p_{\lambda, n}(x)(d x)^{2}}{\left(x^{n}-1\right)\left(x^{n}-\lambda\right)},
$$

with $\operatorname{deg}\left(p_{\lambda, n}\right) \leq 2 n-4$. Moreover, since by Proposition $2.7 \omega_{\lambda, n}$ has to be invariant under the automorphism $(x, y) \rightarrow\left(\xi_{n} x, y\right)$, we see that the actual form of $\omega_{\lambda, n}$ is

$$
\omega_{\lambda, n}(x)=\frac{c_{n}(\lambda) x^{n-2}(d x)^{2}}{\left(x^{n}-1\right)\left(x^{n}-\lambda\right)}
$$

for some uniquely determined constant $c_{n}(\lambda) \in \mathbb{C}$. Thus, we have

Proposition 4.2. The curve $C_{\lambda, n}$ is a Whittaker surface if and only if the constant $c_{n}(\lambda)$ defined by formula (4.1) vanishes. 
Therefore a closer understanding of this function $c_{n}(\lambda)$ is required.

Proposition 4.3. The function $c_{n}(\lambda)$ enjoys the following properties:

i) $c_{n}(\lambda)$ is a real valued function.

ii) $c_{n}(\lambda)$ is analytic.

iii) $c_{n}(1 / \lambda)=c_{n}(\lambda) / \lambda$.

Proof. i) Since we have

$$
c_{n}(\lambda)=\frac{S_{\lambda, n}(z)-W_{\lambda, n}(z)}{z^{n-2} /\left(z^{n}-1\right)\left(z^{n}-\lambda\right)},
$$

it is enough to see that for $z \in \mathbb{R}, S_{\lambda, n}(z)$ is also real.

Now it is known (see [12]) that $S_{\bar{A}}(\bar{z})=\overline{S_{A}(z)}$, where $\bar{A}$ is the set obtained from $A$ by conjugation. In our case, $A_{\lambda, n}$ agrees with its conjugate $\overline{A_{\lambda, n}}$, and the result follows.

ii) This is a consequence of the result of Kra [14] that states that the accessory parameters depend real-analytically on the branching values.

iii) This property can be proved by making $T(x)=1 / x$ in Proposition 2.4 .

The above statement iii) will not be used in the rest of this paper, but we observe that it implies that $c_{n}(-1)=0$, in agreement with the fact that $C_{-1, n}$ is a surface with many automorphisms. We also note that the property stated in iii) carries no information for $\lambda=0$ and $\lambda=1$. This is not surprising, since the function $c_{n}(\lambda)$ is not defined at these points. Nevertheless, one of the key points of this article is the computation of these limit values. In performing that task the main tool will be a powerful result of Kra on the limit of accessory parameters, which we now quote.

4.1. Kra's theorem on the degeneration of accessory parameters. Let $X_{m}$ : $\mathbb{D} \rightarrow \widehat{\mathbb{C}}$ be a sequence of regular covering maps with the set of branch value $A_{m}=$ $\left\{\lambda_{1}^{(m)}, \ldots, \lambda_{n+3}^{(m)}\right\}$, and branching indices $\left(\nu_{1}, \cdots, \nu_{n+3}\right)$.

Assume that $\lim _{m \rightarrow \infty} \lambda_{j}^{(m)}=\lambda_{j}^{(\infty)}$ exists for $j=1, \cdots, n+3$. Denote by $A_{\infty}=\left\{\lambda_{1}^{(\infty)}, \ldots, \lambda_{N}^{\infty}\right\}$ the maximal subset of $\left\{\lambda_{1}^{(\infty)}, \ldots, \lambda_{n+3}^{\infty}\right\}$ that consists of distinct entries, after relabeling if necessary.

Set $\mu_{j}=\nu_{j}$ if a single sequence $\lambda_{j}^{(m)}$ converges to $\lambda_{j}^{(\infty)}$, and $\mu_{j}=\infty$ otherwise. Suppose, moreover, that the signature $\left(0 ; \mu_{1}, \ldots, \mu_{N}\right)$ is hyperbolic.

In this situation, we denote by $X_{\infty}: \mathbb{D} \longrightarrow \widehat{\mathbb{C}}$ the regular cover corresponding to this branching data. The following result holds:

Theorem 4.4 (Kra [14]).

$$
S\left(X_{\infty}^{-1}\right)(z)=\lim _{m \rightarrow \infty} S\left(X_{m}^{-1}\right)(z)
$$

uniformly on compact subsets of $\widehat{\mathbb{C}}$.

4.2. The values $\mathbf{c}_{\mathbf{n}}(\mathbf{0})$ and $\mathbf{c}_{\mathbf{n}}(\mathbf{1})$. We now compute the values of $c_{n}(\lambda)$ as $\lambda \rightarrow 0$ and $\lambda \rightarrow 1$.

Before we start, let us write down the Whittaker rational function for the curve $C_{\lambda, n}$. Replacing in Definition $2.3 f$ by $f_{n}(z)=\left(z^{n}-1\right)\left(z^{n}-\lambda\right)$, we get the following 
expression for $W_{\lambda, n}:=W_{A_{\lambda, n}}$ :

$$
\begin{aligned}
W_{\lambda, n}(z) & =\frac{3 z^{n-2}}{(16 n-8)\left(z^{2 n}-(1+\lambda) z^{n}+\lambda\right)^{2}}\left(\left(2 n^{3}-2 n^{2}\right)(1+\lambda) z^{2 n}\right. \\
& \left.+\left(n^{2} \lambda^{2}+\left(6 n^{2}-8 n^{3}\right) \lambda+n^{2}\right) z^{n}+\left(2 n^{3}-2 n^{2}\right)\left(\lambda+\lambda^{2}\right)\right) .
\end{aligned}
$$

Case $\lambda \rightarrow \mathbf{0}$. In view of Kra's theorem we want to compute

$$
S\left(X_{0, n}^{-1}\right)(z):=\lim _{\lambda \rightarrow 0} S\left(X_{\lambda, n}^{-1}\right)(z),
$$

where $X_{0, n}$ is the regular cover corresponding to the limit branching values, namely $1, \xi, \ldots, \xi^{n-1}, 0$, with branching indices $2, \stackrel{n-1}{.}, 2, \infty$ respectively.

For that purpose, let us consider the map $T: \widehat{\mathbb{C}} \rightarrow \widehat{\mathbb{C}}$ given by $z \rightarrow z^{n}$, and set $Y=T \circ X_{0, n}$. This is clearly a regular cover with only three branch values, namely 0 , 1 and $\infty$. In this case, the classical theory of the Gauss hypergeometric differential equation (see, e.g., [9], p. 303, or [14], p. 593) tells us that the Schwarzian derivative is completely determined by the ramification indices, which in this case are $\infty, 2$ and $n$ respectively. The result is

$$
S\left(Y^{-1}\right)(w)=\frac{\left(\frac{n^{2}-1}{n^{2}}\right) w^{2}+\left(\frac{4-5 n^{2}}{4 n^{2}}\right) w+1}{2 w^{2}(w-1)^{2}} .
$$

On the other hand, direct computation gives

$$
S\left(T^{-1}\right)(w)=\frac{\left(n^{2}-1\right) / 2 n^{2}}{w^{2}}
$$

hence, by equation (2.2) applied to $Y^{-1}=X_{0, n}^{-1} \circ T^{-1}$, one obtains

$$
S\left(Y^{-1}\right)(w)=\frac{1}{n^{2}} w^{(2-2 n) / n} S\left(X_{0, n}^{-1}\right)\left(w^{1 / n}\right)+\frac{\left(n^{2}-1\right) / 2 n^{2}}{w^{2}} .
$$

Therefore

$$
S\left(X_{0, n}^{-1}\right)(z)=\frac{n^{2} z^{2 n-2}\left(\frac{n^{2}-1}{n^{2}} z^{2 n}+\frac{4-5 n^{2}}{4 n^{2}} z^{n}+1\right)}{2 z^{2 n}\left(z^{n}-1\right)^{2}}-\frac{n^{2}-1}{2 z^{2}}=\frac{\frac{3 n^{2}-4}{4} z^{n}+1}{2 z^{2}\left(z^{n}-1\right)^{2}} \text {. }
$$

As for the Whittaker rational function, we see from equation (4.2) that

$$
W_{0, n}(z):=\lim _{\lambda \rightarrow 0} W_{\lambda, n}(z)=\frac{\left(6 n^{3}-6 n^{2}\right) z^{n}+3 n^{2}}{(16 n-8) z^{2}\left(z^{n}-1\right)^{2}} ;
$$

hence

$$
\begin{aligned}
Q_{0, n}(z) & :=S\left(X_{0, n}^{-1}\right)(z)-W_{0, n}(z) \\
& =\frac{\left(\frac{3 n^{2}-4}{4}-\frac{6 n^{3}-6 n^{2}}{8 n-4}\right) z^{n}+1-\frac{3 n^{2}}{8 n-4}}{2 z^{2}\left(z^{n}-1\right)^{2}} \\
& =\frac{\frac{3 n^{2}-8 n+4}{16 n-8}}{z^{2}\left(z^{n}-1\right)} .
\end{aligned}
$$


Therefore, by Theorem 4.4, we have proved

Proposition 4.5. $\lim _{\lambda \rightarrow 0} c_{n}(\lambda)=\frac{3 n^{2}-8 n+4}{16 n-8}$.

Case $\lambda \rightarrow \mathbf{1}$. We proceed as in the previous case.

Now the limit branching data consists of the values $1, \xi, \ldots, \xi^{n-1}$ with branching indices $\infty, \stackrel{n}{.}, \infty$. In order to compute

$$
S\left(X_{1, n}^{-1}\right)(z):=\lim _{\lambda \rightarrow 1} S\left(X_{\lambda, n}^{-1}\right)(z),
$$

we again consider the functions $T(z)=z^{n}$ and $Y=T \circ X_{1, n}$. The branching values of the map $Y$ are again 0,1 and $\infty$, in this case with branching indices $n, \infty, n$ respectively. Therefore

$$
S\left(Y^{-1}\right)(w)=\frac{\frac{n^{2}-1}{n^{2}} w^{2}+\frac{2-n^{2}}{n^{2}} w+\frac{n^{2}-1}{n^{2}}}{2 w^{2}(w-1)^{2}}
$$

and

$$
\begin{aligned}
S\left(X_{1, n}^{-1}\right)(z) & =\frac{\frac{n^{2}-1}{n^{2}} z^{2 n}+\frac{2-n^{2}}{n^{2}} z^{n}+\frac{n^{2}-1}{n^{2}}}{2 z^{2 n}\left(z^{n}-1\right)^{2}} n^{2} z^{2 n-2}+\frac{1-n^{2}}{2 z^{2}} \\
& =\frac{\left(n^{2}-1\right) z^{2 n}+\left(2-n^{2}\right) z^{n}+\left(n^{2}-1\right)}{2 z^{2}\left(z^{n}-1\right)^{2}}+\frac{1-n^{2}}{2 z^{2}} \\
& =\frac{n^{2} z^{n-2}}{2\left(z^{n}-1\right)^{2}} .
\end{aligned}
$$

As for the Whittaker rational function, we have

$$
\begin{aligned}
W_{1, n}(z) & :=\lim _{\lambda \rightarrow 1} W_{\lambda, n}(z) \\
& =3 \frac{\left(4 n^{3}-4 n^{2}\right) z^{3 n-2}+\left(8 n^{2}-8 n^{3}\right) z^{2 n-2}+\left(4 n^{3}-4 n^{2}\right) z^{n-2}}{(16 n-8)\left(z^{n}-1\right)^{4}} \\
& =\frac{\left(12 n^{3}-12 n^{2}\right) z^{n-2}}{(16 n-8)\left(z^{n}-1\right)^{2}} ;
\end{aligned}
$$

hence

$$
Q_{1, n}(z)=S\left(X_{1, n}^{-1}\right)(z)-W_{1, n}(z)=\frac{\left(2 n^{3}-n^{2}\right) z^{n-2}}{(4 n-2)\left(z^{n}-1\right)^{2}} .
$$

Thus we have proved

Proposition 4.6. $\lim _{\lambda \rightarrow 1} c_{n}(\lambda)=\frac{2 n^{2}-n^{3}}{4 n-2}$.

4.3. Conclusion. The study carried out in the previous sections has the following immediate consequence:

Proposition 4.7. Given any $n \geq 3$, there exists at least one value of $\lambda$, say $\lambda_{n} \in(0,1)$, such that the algebraic curve

$$
C_{\lambda_{n}, n}=\left\{y^{2}=\left(x^{n}-1\right)\left(x^{n}-\lambda_{n}\right)\right\}
$$

is a Whittaker surface. 
Proof. As we noted in Proposition 4.3, the function $c_{n}(\lambda)$ is real-analytic, hence continuous. On the other hand, we have just found that

$$
c_{n}(1)=\frac{2 n^{2}-n^{3}}{4 n-2}<0 \quad \text { and } \quad c_{n}(0)=\frac{3 n^{2}-8 n+4}{16 n-8}>0 .
$$

Therefore it has to vanish at some point $\lambda_{n} \in(0,1)$.

In view of Proposition 4.1 we can state the result we have been looking for:

Theorem 4.8. There are Whittaker surfaces which do not have many automorphisms.

\section{AN EXPLICIT NON-WhitTAKER HYPERELLIPTIC SURFACE}

In all branches of mathematics it often occurs that a certain property that, for some theoretical reason, is known to hold generically, turns out to fail whenever one can check it explicitly. There is a good example of this phenomenon inside the theory of Riemann surfaces itself, namely the fact that the generic compact Riemann surface admits no automorphisms (see [17, [7, [1]).

Likewise, we have been unable to locate in the literature a single instance of a hyperelliptic surface without many automorphisms whose accessory parameters can be explicitly computed. The purpose of this last section is to carry out such a computation for the curve $y^{2}=x^{8}+\frac{10}{3} x^{4}+1$. We will find that this surface is non-Whittaker.

Let $C_{1}$ and $C_{2}$ denote the algebraic curves given by $y^{2}=x^{6}-1$ and $y^{2}=$ $x^{8}+\frac{10}{3} x^{4}+1$ respectively. It is not difficult to convince oneself that the morphism

$$
\begin{aligned}
\left\{(x, y): y^{2}=x^{8}+\frac{10}{3} x^{4}+1\right\} & \stackrel{\widetilde{H}}{\longrightarrow}\left\{(x, y): y^{2}=x^{6}-1\right\}, \\
(x, y) & \longmapsto\left(\frac{1+x^{2}}{1-x^{2}}, \frac{2 \sqrt{3} x y}{\left(1-x^{2}\right)^{3}}\right),
\end{aligned}
$$

defines a double covering of $C_{1}$ that is smooth (see [10] for an explicit description of all smooth hyperelliptic double coverings of a given hyperelliptic surface). Moreover, we have the following commutative diagram:

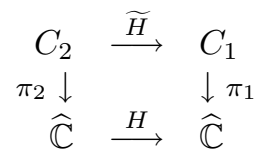

where $H$ is given by $H(x)=\frac{1+x^{2}}{1-x^{2}}$, and $\pi_{i}: C_{i} \longrightarrow \widehat{\mathbb{C}}$ stands for the hyperelliptic map. It follows that, with the notation employed in Definition 2.2, if we denote by $X_{2}: \mathbb{D} \longrightarrow \widehat{\mathbb{C}}$ the regular cover of signature $(0 ; 2, \ldots, 2)$ associated to the curve $C_{2}$, then the one corresponding to $C_{1}$ can be taken to be $X_{1}=H \circ X_{2}$.

Therefore $X_{2}^{-1}=X_{1}^{-1} \circ H$, and hence equation (2.2) yields

$$
S\left(X_{2}^{-1}\right)(x)=S\left(X_{1}^{-1}\right)(H(x))\left(H^{\prime}(x)\right)^{2}+S(H)(x) .
$$

Now, since $C_{1}$ is a surface with many automorphisms, $S\left(X_{1}^{-1}\right)$ is given by the corresponding Whittaker rational function. As for the term $S(H)$, it may be computed directly. 
Therefore, an easy calculation gives the following result.

Proposition 5.1. The Schwarzian derivative $S\left(X_{2}^{-1}\right)(x)$ that corresponds to the hyperelliptic curve $C_{2}=\left\{(x, y): y^{2}=x^{8}+\frac{10}{3} x^{4}+1\right\}$ is given by

$$
S\left(X_{2}^{-1}\right)(x)=\frac{-48\left(3 x^{8}+2 x^{4}+3\right) x^{2}}{\left(3 x^{8}+10 x^{4}+3\right)^{2}} .
$$

As for the Whittaker rational function of $C_{2}$, we find that

$$
W_{2}(x)=-\frac{24}{7} \frac{x^{2}\left(38 x^{4}+45 x^{8}+45\right)}{\left(10 x^{4}+3+3 x^{8}\right)^{2}} .
$$

Since $W_{2}$ does not coincide with the expression obtained in Proposition 5.1 we get

Corollary 5.2. $C_{2}$ is a non-Whittaker surface.

Nevertheless, the accessory parameters of $C_{2}$ can be explicitly computed. In fact, with the notation introduced in equation (2.4), we have

Corollary 5.3. The accessory parameters of the normal cover $X_{2}: \mathbb{D} \longrightarrow \widehat{\mathbb{C}}$ corresponding to the curve $C_{2}$ are $c_{6}=-8, c_{2}=-\frac{8}{3}$, and $c_{j}=0$ if $j \neq 2,6$.

Proof. All we have to do is to notice that $S\left(X_{2}^{-1}\right)$ can be rewritten in the form

$$
S\left(X_{2}^{-1}\right)(x)=\frac{3}{8}\left(\sum_{i=1}^{8} \frac{1}{\left(x-a_{i}\right)^{2}}+\frac{-8 x^{6}-\frac{8}{3} x^{2}}{x^{8}+\frac{10}{3} x^{4}+1}\right),
$$

where $a_{1}, \ldots, a_{8}$ are the eight roots of $x^{8}+\frac{10}{3} x^{4}+1$.

\section{REFERENCES}

1. R. Brandt, H. Stichtenoth, Die Automorphismengruppen hyperelliptischer Kurven. Manuscripta Math. 55 (1986), 83-92. MR 87m:14033

2. R. Brooks, H.M. Farkas and I. Kra, Number theory, theta identities, and modular curves. Contemporary Mathematics 201. AMS. MR 98m:11030

3. E. Bujalance, J.M. Gamboa, G. Gromadzki, The full automorphism group of hyperelliptic Riemann surfaces. Manuscripta Math. 79 (1993), 267-282. MR 94f:20093

4. D.V. Chudnovsky and G.V. Chudnovsky, Computer algebra in the service of mathematical physics and number theory. Lecture Notes in Pure and Applied Mathematics (1990). Dekker. MR 92g:11122

5. D.P. Dalzell, A note on automorphic functions. J. London Math. Soc. 5 (1930), 280-282.

6. S.C. Dhar, On the uniformization of a special kind of algebraic curves of any genus. J. London Math. Soc. 10 (1935), 259-263.

7. B. Everitt, A family of conformally asymmetric Riemann surfaces. Glasgow Math. J. 39 (1997), 221-225. MR 98i:30055

8. H.M. Farkas, I. Kra, Riemann surfaces. Springer-Verlag (1992). MR 93a:30047

9. L.R. Ford, Automorphic functions. Chelsea Publishing Company (1951).

10. Y. Fuertes, G. González-Diez, Smooth hyperelliptic double coverings of hyperelliptic surfaces. Preprint.

11. E. Girondo, G. González-Diez, On extremal Riemann surfaces and their uniformizing Fuchsian groups. Glasgow Math. J. 44 (2002), 149-157. MR 2002m:30053

12. J.A. Hempel, On the uniformization of the n-punctured sphere. Bull. London Math. Soc. 20 (1988), 97-115. MR 89c:30109

13. J. Hodgkinson, Note on the uniformization of hyperelliptic curves. J. London Math. Soc. 11 (1936), 185-192.

14. I. Kra, Accesory parameters for punctured spheres. Transactions of the A.M.S. vol. 313 (1989), 589-617. MR 89j:30062 
15. M. Mursi, On the uniformisation of algebraic curves of genus 3. Proc. Edinburgh Math. Soc. 2 (1930), 101-107.

16. R.A. Rankin, The differential equations associated with the uniformization of certain algebraic curves. Proc. Roy. Soc. Edinburgh Sect. A 65 (1958), 35-62. MR 19:1172d

17. P. Turbek, An explicit family of curves with trivial automorphism group. Proc. Amer. Math. Soc. 122 (1994), 657-664. MR 95a:14034

18. E.T. Whittaker, On the connexion of algebraic functions with automorphic functions. Phil. Trans. Roy. Soc. London 192A (1899), 1-32.

19. E.T. Whittaker, On hyperlemniscate functions. A family of automorphic functions. J. London Math. Soc. 4 (1929), 274-278.

20. J.M. Whittaker, The uniformisation of algebraic curves. J. London Math. Soc. 5 (1930), $150-154$.

21. E.T. Whittaker, G.N. Watson, A course of modern analysis. 4th. ed., Cambridge U. Press (1927). MR 31:2375 (1962 reprint)

Departamento de Matemáticas, Universidad Autónoma de Madrid, Madrid 28049, SPAIN

E-mail address: ernesto.girondo@uam.es

Departamento de Matemáticas, Universidad Autónoma de Madrid, Madrid 28049, SPAIN

E-mail address: gabino.gonzalez@uam.es 